\title{
An Effective Proof that Open Sets are Ramsey
}

\author{
Jeremy Avigad
}

January 22, 1996

\begin{abstract}
Solovay has shown that if $\mathcal{O}$ is an open subset of $P(\omega)$ with code $S$ and no infinite set avoids $\mathcal{O}$, then there is an infinite set hyperarithmetic in $S$ that lands in $\mathcal{O}$. We provide a direct proof of this theorem that is easily formalizable in $A T R_{0}$.
\end{abstract}

\section{Introduction}

A plausible generalization of Ramsey's theorem asserts that for every twocoloring of the infinite subsets of $\omega$ there is an infinite homogeneous set, that is, an infinite subset of $\omega$ every infinite subset of which has been assigned the same color. Unfortunately, under the axiom of choice, this generalization is false: by transfinite recursion along a well-ordering of the reals one can cook up a coloring with no infinite homogeneous set. On the other hand, the nonconstructive nature of this counterexample suggests that perhaps the theorem might hold true for colorings that are "well-behaved" or "easily definable."

To that end, we define a partition to be a subset of the power set of $\omega$, with the understanding that the infinite subsets falling inside the partition are colored, say, red, and those outside the partition are colored blue. If $\mathcal{P}$ is a partition and $X$ is an infinite subset of $\omega$, then $X$ lands in $\mathcal{P}$ if every infinite subset of $X$ is in $\mathcal{P}$, and $X$ avoids $\mathcal{P}$ if no infinite subset of $X$ is in $\mathcal{P}$. A partition $\mathcal{P}$ is Ramsey if there is an infinite set $X$ that either lands in $\mathcal{P}$ or avoids $\mathcal{P}$. The theorems we are interested in are of the form "every well-behaved partition is Ramsey." A number of authors have shown independently that if $\mathcal{P}$ is open in the usual topology then it is Ramsey (see [4]), and the conclusion has been extended to Borel sets by Galvin and Prikry [4] and analytic sets by Silver $[8,2]$.

Solovay [11] has strengthened the result for open sets as follows: if $\mathcal{O}$ is an open set with code $S$ and no infinite set avoids $\mathcal{O}$, then there is an infinite set hyperarithmetic in $S$ which lands in $\mathcal{O}$. Mansfield [7] has provided a shorter proof of this theorem that was used in [3] to show that the subsystem of secondorder arithmetic $A T R_{0}$ proves (and is in fact over a weak base theory equivalent 
to) Solovay's result. The formalization of Mansfield's proof in $A T R_{0}$ is, however, somewhat difficult.

Below we present a remarkably direct proof of Solovay's theorem, obtained by "effectivizing" an argument that uses a nonprincipal ultrafilter on $\omega$. Our proof is easily formalizable in $A T R_{0}$. For more elaborate uses of ultrafilter methods in proving Ramsey-theoretic statements see $[6,5,1]$, and for more information on $A T R_{0}$ and other subsystems of second-order arithmetic see, for example, $[3,9,10]$.

I'd like to thank Andreas Blass for showing me the ultrafilter proof in Section 2 and suggesting the use of Lemma 3.2, and Stephen Simpson for helpful comments on a draft of this paper. The effective proof of Solovay's theorem appears in Section 3.

\section{The noneffective version}

From now on we identify finite and infinite subsets of $\omega$ with the sequences that enumerate their elements in increasing order. Let $T$ be the tree of finite increasing sequences from $\omega$, and let the variables $\alpha, \beta, \sigma, \tau$ denote elements of $T$. The notation $\sigma \subseteq \tau$ means that (the set associated with) $\sigma$ is a subset of (the set associated with) $\tau$ and not necessarily that $\sigma$ is an initial segment of $\tau$.

A basis for the usual topology on $P(\omega)$ is given by sets of the form

$$
\mathcal{B}_{\sigma}=\{X \mid X \text { extends } \sigma\},
$$

and a set of sequences $S$ can be taken to code the open set

$$
\mathcal{O}=\bigcup_{\sigma \in S} \mathcal{B}_{\sigma} .
$$

Though the assignment of codes to open sets is not unique, it is well known that a set $\mathcal{O}$ is $\Sigma_{1}^{0}$ definable from a parameter $A$ if and only if $\mathcal{O}$ is open and has a code recursive in $A$.

Theorem 2.1 Open sets are Ramsey.

Proof. Let $\mathcal{O}$ be an open subset of $P(\omega)$ with code $S$. Without loss of generality we can assume that $S$ is closed under extensions, since otherwise the set

$$
S^{\prime}=\{\sigma \mid \text { some initial segment of } \sigma \text { is in } S\}
$$

also codes $\mathcal{O}$ and has this property. Fix $\mathcal{U}$, a nonprincipal ultrafilter on $\omega$.

By transfinite recursion on the ordinals we label certain elements $\sigma$ of $T$ good and associate an element $U_{\sigma}$ of $\mathcal{U}$. At stage 0 , we label a sequence $\sigma$ good if $\sigma$ is in $S$, and set $U_{\sigma}=\omega$. At stage $\mu$ we label $\sigma \operatorname{good}$ if $\sigma$ has not already 
been so labelled and the set of elements $n$ such that $\sigma^{\wedge} n$ is good is in $\mathcal{U}$. In this case we set

$$
U_{\sigma}=\{n \mid \hat{\sigma} n \text { was labelled good before stage } \mu\} .
$$

Since $T$ is countable, this process stabilizes at some stage before $\omega_{1}$. At this point label the remaining elements $\sigma$ of $T$ bad and set

$$
U_{\sigma}=\left\{n \mid \hat{\sigma^{\wedge}} n \text { is } \operatorname{bad}\right\} .
$$

Note that if $\sigma$ is bad then $U_{\sigma}$ is in $\mathcal{U}$, since otherwise its complement would be in $\mathcal{U}$ and we would have labelled $\sigma$ good.

We claim that if the empty sequence is bad, there is a set which avoids $\mathcal{O}$, and if empty sequence is good, there is a set which lands in $\mathcal{O}$.

Suppose the empty sequence is bad. We construct an increasing sequence $x_{0}, x_{1}, x_{2}, \ldots$ every subsequence of which is bad. Take $x_{0}$ to be any element of $U_{\langle\rangle}$. Once $x_{0}, x_{1}, \ldots, x_{n}$ have been chosen, note that the set

$$
\bigcap_{\sigma \subseteq\left\langle x_{0}, x_{1}, \ldots, x_{n}\right\rangle} U_{\sigma}
$$

is in $\mathcal{U}$, and so we can take $x_{n+1}$ to be any element of this set that is greater than $x_{n}$.

Let $X=\left\langle x_{0}, x_{1}, x_{2}, \ldots\right\rangle$. This set $X$ avoids $\mathcal{O}$ : if some $Y \subseteq X$ were an element of $\mathcal{O}$, we'd have a sequence $\left\langle y_{0}, y_{1}, \ldots, y_{n}\right\rangle \subseteq X$ in $S$. But this sequence would have been labelled good at stage 0 , contradicting the fact that every subsequence of $X$ is bad.

So now suppose the empty sequence is good. Exactly as before, construct an increasing sequence $x_{0}, x_{1}, x_{2}, \ldots$ every subsequence of which is good. Let $X=$ $\left\langle x_{0}, x_{1}, x_{2}, \ldots\right\rangle$. We claim that $X$ lands in $\mathcal{O}$. Let $Y=\left\langle y_{0}, y_{1}, y_{2}, \ldots\right\rangle$ be any infinite subset of $X$, and for each $n$ let $\mu_{n}$ be the stage at which $\left\langle y_{0}, y_{1}, \ldots, y_{n}\right\rangle$ was labelled good. Then if $\mu_{n} \neq 0$ we have that $\mu_{n+1}<\mu_{n}$, since $y_{n+1}$ is in $U_{\left\langle y_{0}, y_{1}, \ldots, y_{n}\right\rangle}$ and $\left\langle y_{0}, y_{1}, \ldots, y_{n}\right\rangle$ was labelled good by virtue of this set. Since any descending sequence of ordinals must eventually hit 0 , we will have $\mu_{m}=0$ for some $m$, in which case $\left\langle y_{0}, y_{1}, \ldots, y_{m}\right\rangle \in S$ and hence $Y \in \mathcal{O}$.

\section{The effective version}

Making the foregoing argument more effective involves two observations:

1 . We don't need the entire ultrafilter $\mathcal{U}$; it is enough to keep track of countably many sets that we've committed to being in the ultrafilter.

2. We don't need the entire tree $T$. On the assumption that no set avoids $\mathcal{O}$, we can restrict our attention to a well-founded subtree $T^{\prime}$, and then label the nodes "from the bottom up." 
Theorem 3.1 Let $\mathcal{O}$ be an open subset of $P(\omega)$ with code $S$, and suppose no infinite $X$ avoids $\mathcal{O}$. Then there is an infinite $X$ hyperarithmetic in $S$, such that $X$ lands in $\mathcal{O}$.

Proof. Fix $\mathcal{O}$ and $S$ as in the hypothesis of the theorem, and suppose no infinite $X$ avoids $\mathcal{O}$. Let

$$
T^{\prime}=\{\sigma \mid \text { no subsequence of } \sigma \text { is in } S\}
$$

and note that $T^{\prime}$ is a tree that is closed under subsequences. We claim $T^{\prime}$ is wellfounded: Since no infinite $X$ avoids $\mathcal{O}$, every infinite $X$ has a finite subsequence $\sigma$ in $S$. But no such $X$ can be a path through $T^{\prime}$.

We start by labelling sequences outside of $T^{\prime}$ either good or bad. If $\sigma$ is outside of $T^{\prime}$, let $\tau$ be the smallest initial segment of $\sigma$ that is outside of $T^{\prime}$. If $\tau$ is in $S$ we label $\sigma$ good, and otherwise we label $\sigma$ bad.

Recall the Brouwer-Kleene ordering on $T^{\prime}$, in which $\sigma \prec \tau$ iff $\sigma$ extends $\tau$ or $\sigma$ is less than $\tau$ in the lexicographical ordering. Since $T^{\prime}$ is well-founded, $\prec$ is a well-ordering. Our construction proceeds by transfinite recursion along $\prec$, where at stage $\alpha$ we label the node $\alpha$ good or bad and at the same time define a set $U_{\alpha}$, so that the following hold:

1. Each $U_{\alpha}$ is infinite.

2. If $\alpha \succ \beta$ then $U_{\alpha} \subseteq_{f} U_{\beta}$, i.e. $U_{\alpha} \backslash U_{\beta}$ is finite.

3. If $\alpha$ is good then for all $n \in U_{\alpha}, \alpha \hat{n}$ is good.

4. If $\alpha$ is bad then for all $n \in U_{\alpha}, \alpha \hat{n}$ is bad.

We will need to use the following

Lemma 3.2 Suppose for each $\beta \prec \alpha$ we've chosen $U_{\beta}$ so that clauses (1) and (2) hold. Then there is an infinite set $Z$ such that for every $\beta \prec \alpha$ we have $Z \subseteq_{f} U_{\beta}$.

Proof. If $\alpha$ is the least element in the ordering we can take $Z=\omega$, and if $\alpha$ is the successor of $\beta$ we can take $Z=U_{\beta}$. In the case where $\alpha$ is a limit, we take a diagonal intersection: since there are only countably many $\beta \prec \alpha$ we can find a countable sequence $\beta_{i}$ cofinal in $\alpha$. Take $u_{0}$ to be the least element in $U_{\beta_{0}}$, and take $u_{i+1}$ to be the least element in

$$
\bigcap_{j \leq i}\left(U_{\beta_{j}} \backslash\left\{u_{j}\right\}\right)
$$

It is straightforward to verify that $Z=\left\{u_{0}, u_{1}, u_{2} \ldots\right\}$ has the desired property.

We now describe the construction. Suppose we've constructed $U_{\beta}$ for all $\beta \prec \alpha$ and labelled each node $\beta \prec \alpha$ good or bad, so that clauses (1)-(4) hold. 
At stage $\alpha$, first use the lemma to pick an infinite $Z$ so that for all $\beta \prec \alpha$, $Z \subseteq_{f} U_{\beta}$. Then consider

$$
W=\left\{n \in Z \mid \sigma^{\wedge} n \text { is good }\right\} .
$$

If $W$ is infinite, label $\alpha$ good and take $U_{\alpha}=W$. Otherwise label $\alpha$ bad and take $U_{\alpha}=Z \backslash W$. The process continues until the empty sequence (i.e. the root of $T$ ) has been labelled and $U_{\langle\rangle}$has been defined.

Now define $U_{\sigma}=\omega$ for all $\sigma$ outside of $T^{\prime}$, and note that clauses (3) and (4) still hold for such $\sigma$.

We claim that the empty sequence is good. To prove the claim, suppose the empty sequence were bad. We build an increasing sequence of elements $x_{0}, x_{1}, x_{2}, \ldots$, every subsequence of which is bad. Let $x_{0}$ be any element of $U_{\langle\rangle}$ and once $x_{0}, x_{1}, \ldots, x_{n}$ have been chosen, let

$$
U=\bigcap_{\sigma \subseteq\left\langle x_{0}, \ldots, x_{n}\right\rangle} U_{\sigma} .
$$

Since $U_{\langle\rangle} \subseteq_{f} U_{\sigma}$ for each of these (finitely many) $\sigma$, we have $U_{\langle\rangle} \subseteq_{f} U$, and hence $U$ is infinite. Take $x_{n+1}$ to be any (e.g. the least) element of $U$ that is greater than $x_{n}$.

Let $X=\left\{x_{0}, x_{1}, x_{2}, \ldots\right\}$. Since we're assuming that no infinite set avoids $\mathcal{O}$, some subsequence $\sigma$ of $X$ is in $S$. Take $\sigma$ minimal, so that no proper subsequence of $\sigma$ is in $S$. Then $\sigma$ is outside of $T^{\prime}$ and every initial segment of $\sigma$ is in $T^{\prime}$. But we initially labelled such $\sigma$ good, contradiction. This proves our claim that the empty sequence is good.

Now use the same construction to obtain an increasing sequence $x_{0}, x_{1}, x_{2}, \ldots$ every subsequence of which is good. Let $X=\left\{x_{0}, x_{1}, x_{2}, \ldots\right\}$. We claim that $X$ lands in $O$. Let $Y$ be any infinite subset of $X$. Since $T^{\prime}$ is well-founded, there is a smallest initial segment $\sigma$ of $Y$ that is outside of $T$. By our construction of $X$ we know that $\sigma$ is good, and hence $\sigma$ is in $S$. So $Y$ is in $O$, proving our claim.

Since the ordering $\prec$ is recursive in $S$, and for each $\sigma$ in $T^{\prime}$ the set $U_{\sigma}$ is arithmetically definable from $S$ and the sequence $\left\langle U_{\tau}\right\rangle_{\tau \prec \sigma}$, it is easy to verify that $X$ is hyperarithmetic in $S$.

Corollary 3.3 ATR $R_{0}$ proves Theorem 3.1 (and hence the fact that open sets are Ramsey).

Proof. Formalizing the above argument in $A T R_{0}$ is straightforward (see $[3,9$, 10]).

\section{References}

[1] Blass, Andreas, "Selective ultrafilters and homogeneity," Annals of Pure and Applied Logic, vol. 38 (1988), 215-255. 
[2] Ellentuck, Erik, "A new proof that analytic sets are Ramsey," Journal of Symbolic Logic, vol. 39 (1974), 163-165.

[3] Friedman, Harvey, Kenneth McAloon, and Stephen Simpson, "A finite combinatorial principle which is equivalent to the 1-consistency of predicative analysis," in Patras Logic Symposium, G. Metakides ed., North-Holland, 1982.

[4] Galvin, Fred and Karel Prikry, "Borel sets and Ramsey's Theorem," Journal of Symbolic Logic, vol. 38 (1973), 193-198.

[5] Louveau, A., "Une methode topologique pour l'etude de la propriete de Ramsey," Israel Journal of Mathematics, vol. 23 (1976), 97-116.

[6] Mathias, A., "Happy families," Annals of Mathematical Logic, vol. 12 (1977), 59-111.

[7] Mansfield, Richard, "A footnote to a theorem of Solovay on recursive encodability," in Macintyre et al. eds., Logic Colloqium 'rn, North-Holland (1978), 195-198.

[8] Silver, Jack, "Every analytic set is Ramsey," Journal of Symbolic Logic, vol. 35 , no. 1 (1970), 60-64.

[9] Simpson, Stephen, "Friedman's research on subsystems of second-order arithmetic," in Harrington et al. eds., Harvey Friedman's Research on the Foundations of Mathematics, North Holland (1985).

[10] Simpson, Stephen, Subsystems of Second Order Arithmetic, preprint.

[11] Solovay, Robert, "Hyperarithmetically encodable sets," Transactions of the $A M S$, vol. 239 (1978), 99-122. 